A CALCIUM OXIDE SORBENT PROCESS

FOR BULK SEPARATION OF CARBON DIOXIDE

Contract No. DE-AC21-89MC26366

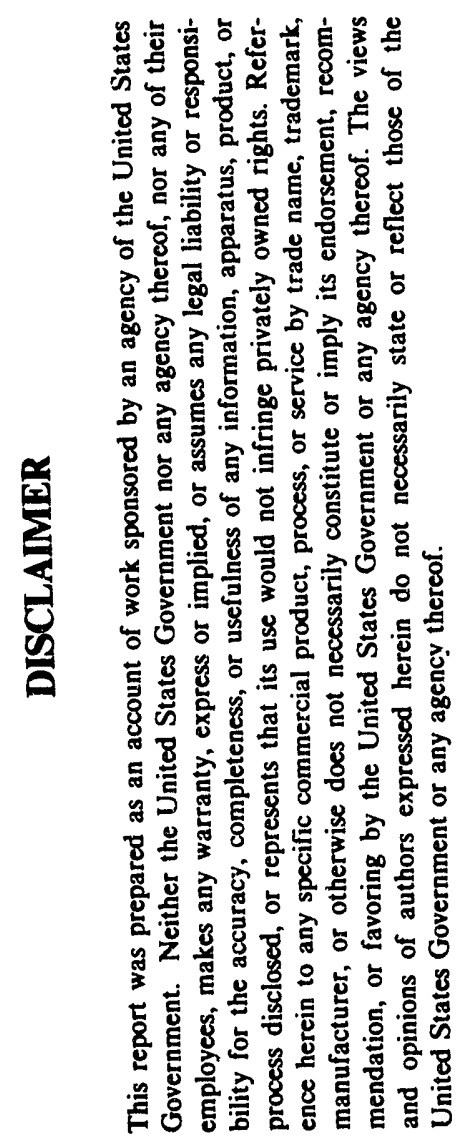

Quarterly Progress Report 13

October 1992 - December 1992

\author{
Department of Chemical Engineering \\ Louisiana State University \\ Baton Rouge, Louisiana 70803
}

(504) 388-1426

Dougias $P$. Harrison
Principal Investigator

January 1993
$\mathrm{DOE} / \mathrm{MC} / 26366--3304$

DE93 009651

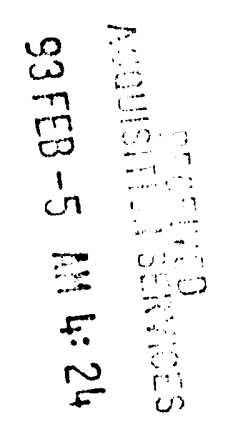


EXECUTIVE SUMMARY

This research project is investigating the technical feasibility of a high-temperature, high-pressure (HTHP) process for the bulk separation of $\mathrm{CO}_{2}$ from coal-derived gas. The oiliginal contract for two years was awarded in September 1989 as a result of solicitation number DE-RA21-89MC26040 entitled "Novel Concepts for Bulk Separation of Gases in Coal Gasification Systems." Two no-cost extensions without a change in the statement of work extended the performance period by six months to March 1992. At that time a two-year contract extension with an enlarged scope of work and additional funding was approved so that the research is now scheduled to end in March 1994.

Phase I research, in which an electrobalance reactor was used to establish the technical feasibility of the regenerable sorbent process, was completed in March 1992 and results have been fully described in earlier quarterly reports. In Phase $I$, the calcination and carbonation characteristics of three calcium sorbents were studied as a function of calcination and carbonation temperature and pressure, mol fraction $\mathrm{CO}_{2}$ in the carbonation gas, and carbonation background gas composition. Desirable reaction conditions $r . j u$ ured for high reactivity and good sorbent durability were determined. Multicycle tests consisting of as many as ten complete calcination and carbonation cycles were completed. Indirect evidence which suggested that the water-gas shift reaction occurred simultaneously with $\mathrm{CO}_{2}$ removal was found. Occurrence of the simultaneous reactions created the possibility of a direct one-step process for the manufacture of hydrogen from coal-gas while at the same time separating a concentrated stream of $\mathrm{CO}_{2}$. The concentrated $\mathrm{CO}_{2}$ stream could be quite significant if, in the future, environmental. regulations restrict atmospheric $\mathrm{CO}_{2}$ emissions. 
Phase II research involves a scale-up from microgram to gram quantities of sorbent and a switch from the electrobalance reactor to a fixed-bed reactor with capability for feed and product gas analysis. At the end of the previous quarter, essentially all major components of the reactor system had been received. Assembly and testing of the system were completed during the present quarter, and a first reaction test involving calcination of dolomite was carried out. Much of the emphasis during the quarter was devoted to developing the gas chromatograph analytical procedure and calibrating the chromatograph using standard gas mixtures of known composition. Continuing problems involving plugging of the nickel-catalyzed methanizer which seemed to occur when the sample contained high concentrations of carbon oxides ( $\mathrm{CO}$ and $\mathrm{CO}_{2}$ ) led to the development of an alternate analytical method which utilizes only the thermal conductivity detector. This new method is simpler since neither the methanizer or flame ionization detector are required, but the sensitivity for $\mathrm{CO}$ and $\mathrm{CO}_{2}$ is less than desired.

Three topics are covered in this quarterly report. First, the current status of the reactor system and the series of preliminary tests leading to the first reaction test are described. The second topic describes our effort to develop the gas chromatograph analytical method and to calibrate the gas chromatograph using gas mixtures of known composition. The third topic describes results of the first reaction test in which dolomite (sorbent 9) was calcined at $750^{\circ} \mathrm{C}$ and $15 \mathrm{~atm}$ in flowing $\mathrm{N}_{2}$. 
FIXED-BED REACTON ASSEMBLY AND TESTING

The final components of the fised-bed reactor system were received permitting assembly to be completed and testing to be initiated. A diagram of the present reactor system is presented in Figure 1 while the reactor itself is shown in Figure 2. These figures are similar to the figures included in the previous quarterly report, but include modifications which have been made during the past quarter. These design changes are described in the following.

1. The mol sieve filter shown previously in the reactor product line and bypass feed lines (Figure 1) has been removed. Our analytical calibration tests have indicated the possibility of a significant sink/source of $\mathrm{CO}_{2}$ in the system. Since $\mathrm{CO}_{2}$ is known to be strongly adsorbed on mol sieves, the filters could have served as a $\mathrm{CO}_{2}$ sink under flow conditions involving high $\mathrm{CO}_{2}$ concentrations, and as a $\mathrm{CO}_{2}$ source when the $\mathrm{CO}_{2}$ concentration in the flowing gas was subsequently decreased.

2. Figure 2 shows only two porous stainless steel disks, one at the top and one at the bottom of the packed bed. The previous design also included three intermediate disks which separated the sorbent bed into four axial sections. We determined experimentally that five disks made it extremely difficult to load and unload the reactor. Removing the intermediate disks eases the loading/unloading problem, and we believe it wıll still be possible to separate the sorbent into four axial sections for sorbent characterization at the conclusion of a run. In addition, the quartz wool packing previously shown at the bottom of the sorbent bed has been eliminated. This 


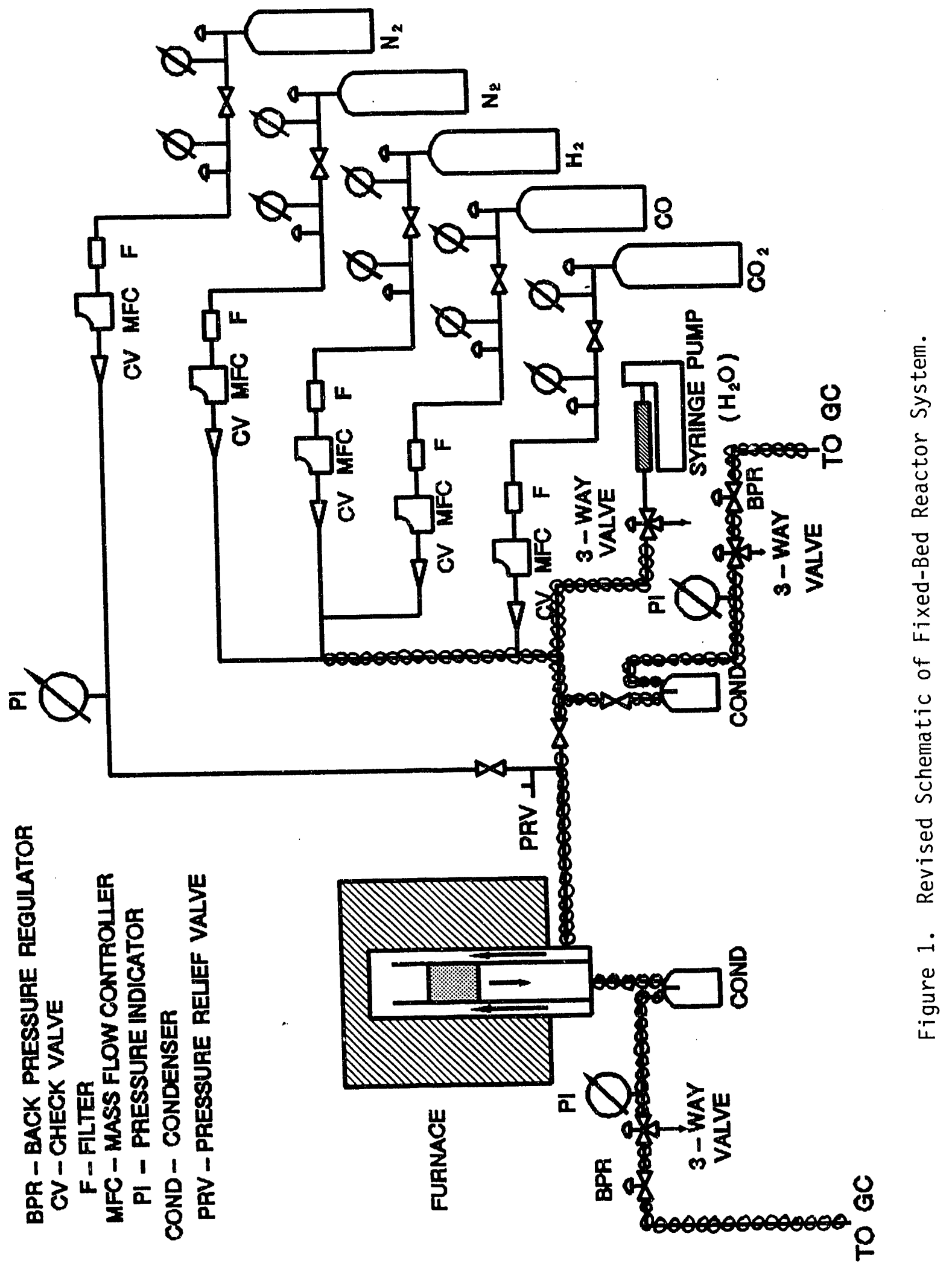




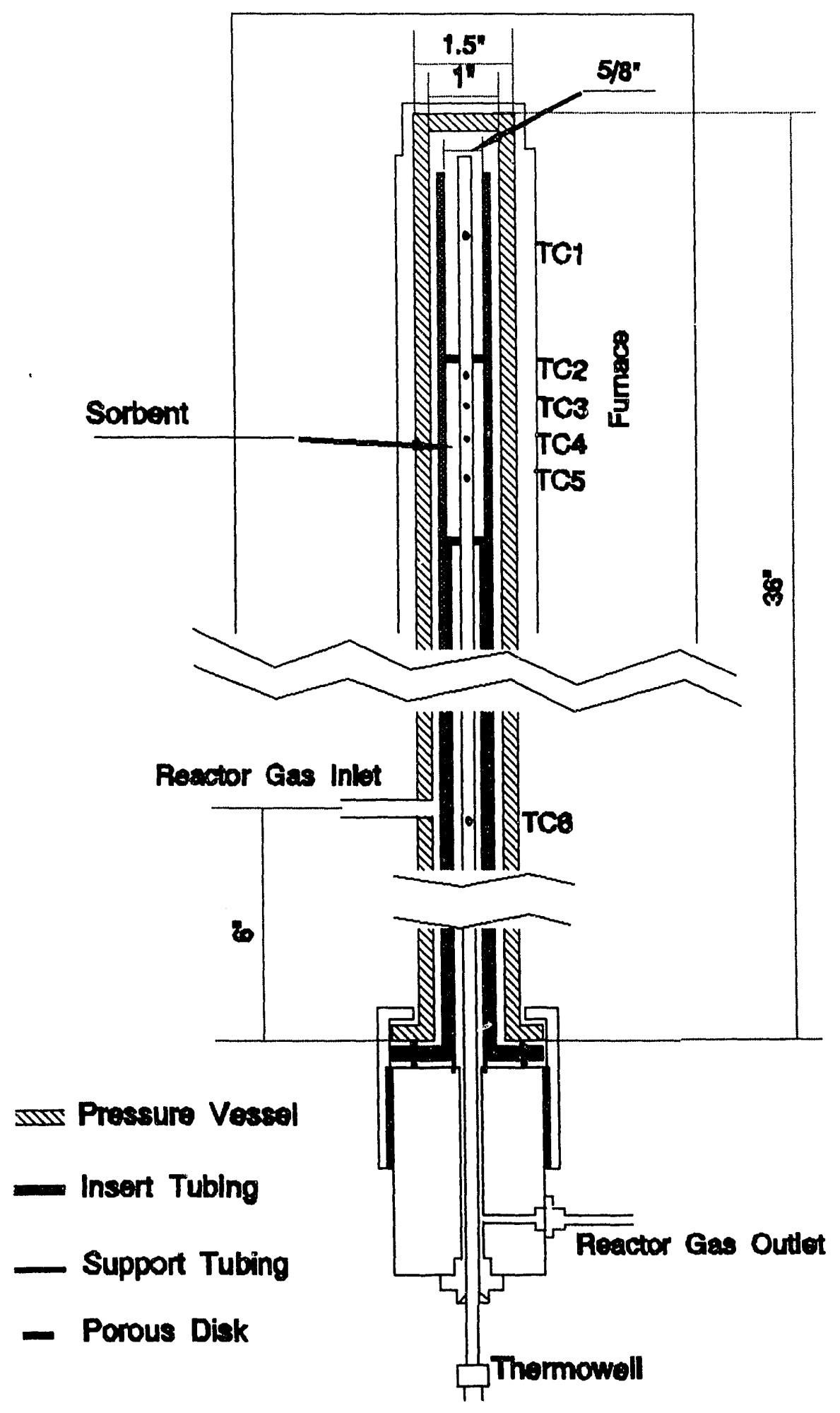

Reactor System

Figure 2. Revised Schematic of Fixed-Bed Reactor. 
change increases the amount of sorbent which may be used by approximately $20 \%$.

The final significant component of the reactor system to be delivered was the thermowell containing the six axially spaced thermocouples (Figure 2). This was a specially fabricated item which had to be returned to the fabricator twice for corrections. Once the reactor system was assembled, room temperature leaks were identified and corrected. Then a series of pressure tests involving progressively higher temperatures was initiated. At the end of each temperature test the reactor was disassembed and examined visually. Two critical high temperature parts in the reactor are the o-ring which provides the pressure seal and the porous stainless disks used to support the sorbent bed. As previously mentioned the number of disks has been reduced from five to two.

No problems were observed until a set-point temperature of $500^{\circ} \mathrm{C}$ was reached. After that test it was difficult to remove the stainless disks from the reactor insert. The problem was attributed to uneven thermal expansion. As a consequence the disks were remachined with the outer diameter of the disk reduced by 0.003 inches and the diameter of the hole in the center of the disks increased by 0.003 inches. By changing the dimensions and reducing the number of disks from five to two it is possible to load and unluad the reactor if sufficient care and patience are used.

At this time the reactor has been heated to a maximum of $800^{\circ} \mathrm{C}$ under flowing $\mathrm{N}_{2}$ at $15 \mathrm{~atm}$. No problems with overheating of the 0-ring have been encountered.

Figures 3 and 4 show the temperature distributions at the various thermocouple positions in a test where the set-point temperature was 


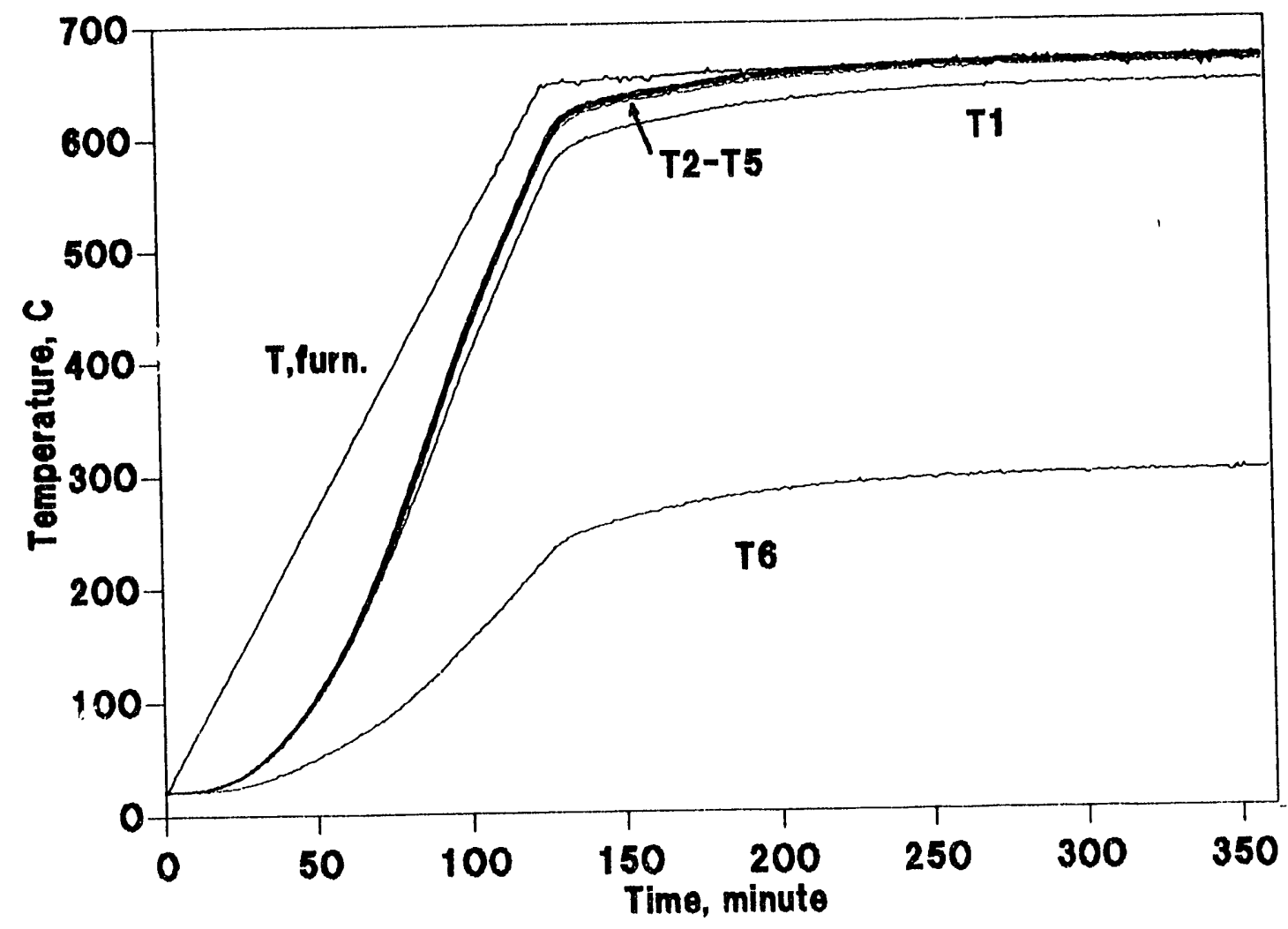

Figure 3. Reactor Temperature-Time Response in Nonreacting Experimental Test. 


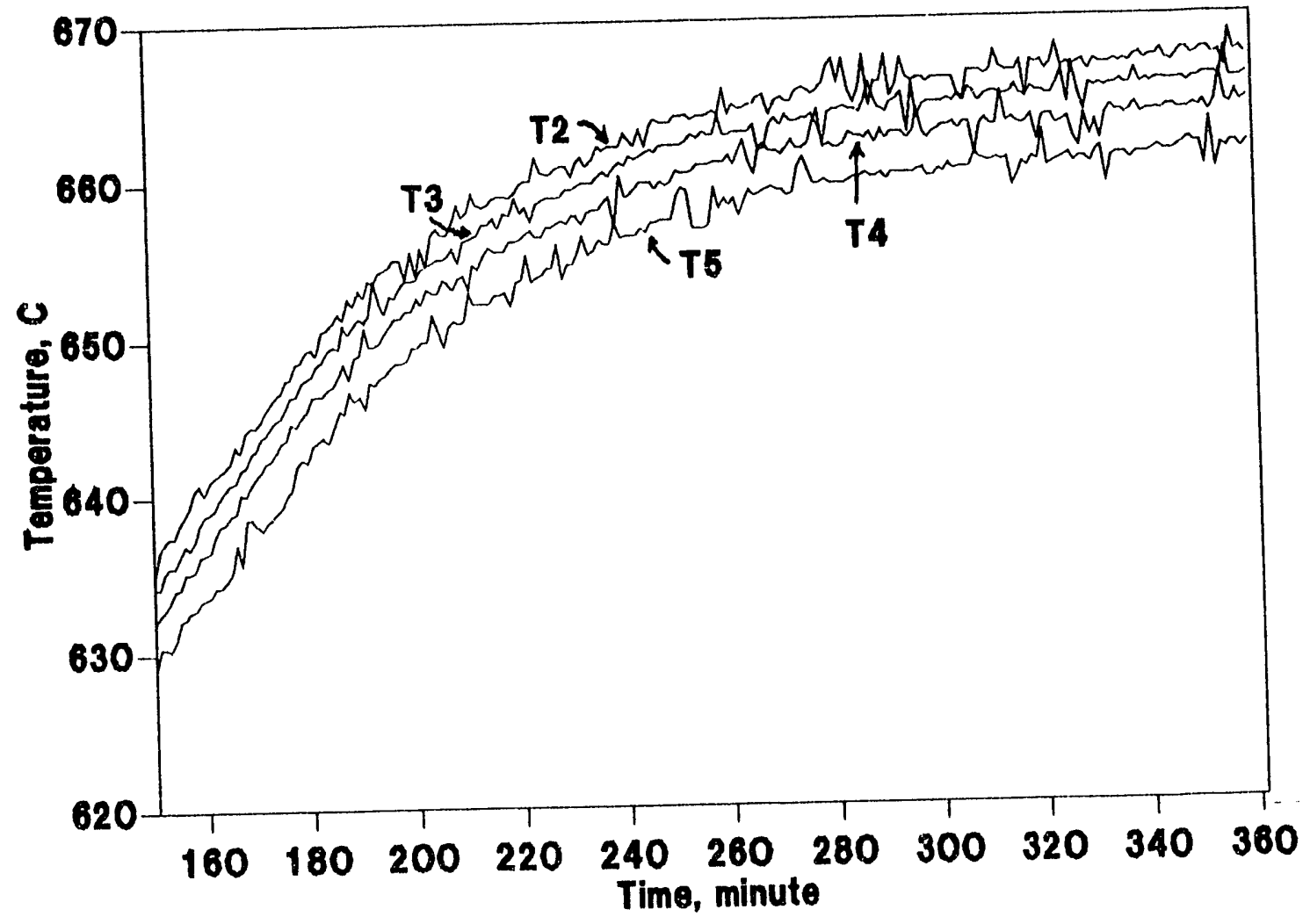

Figure 4. Enlargement of Figure 3 Showing Axial Temperatures Within the Bed (Nonreacting Test). 
increased at $5^{\circ} \mathrm{C} / \mathrm{min}$ to $660^{\circ} \mathrm{C}$ and held constant thereafter. In Figure 3, $T_{\text {furn }}$ represents the furnace temperature. $\mathrm{T}_{1}$ represents the temperature of the preheated feed gas; $\mathrm{T}_{2}$ through $\mathrm{T}_{4}$ represent the temperatures at four axial position in the sorbent bed. $\mathrm{T}_{6}$ represents the temperature of the product gas after heat exchange with the feed gas (see Figure 2).

During the early heating stages there is considerable lag between the set-point and the reactor temperatures. This lag is due to the large mass of stainless steel and is qualitatively similar to the lag observed in the earlier electrobalance tests. Most of the lag was overcome by the time the set point temperature was reached. After approximately 250 minutes the temperature of the preheated feed gas was within about $25^{\circ} \mathrm{C}$ of the set-point temperature, and the temperatures within the bed were, on the Figure 3 scale, approximately equal to each other and also equal to the set point temperature. The temperature of the cooled product gas leveled out at about $300^{\circ} \mathrm{C}$, thus providing an adequate margin of temperature safety for the 0 -ring.

Figure 4 shows the reactor temperatures, $\mathrm{T}_{2}$ through $\mathrm{T}_{5}$ on an enlarged scale during the latter stages of the test. From the figure we see that an axial temperature gradient of appoximately $6^{\circ} \mathrm{C}$ exists, with the temperatures ranging from $668^{\circ} \mathrm{C}$ near the top of the packed bed to $662^{\circ} \mathrm{C}$ near the bottom.

Other pressure-temperature tests under non reaction conditions produced similar results; one test under reaction conditions (to be described later) was completed during the quarter. 


\section{CHROMATOGRAPHIC ANALYSIS}

Much of the effort during the quarter was devoted to developing the proper analytical technique and calibrating the gas chromatograph. Original plans called for the use of thermal conductivity (TCD) and flame ionization (FID) detectors using nitrogen carrier gas. $\mathrm{H}_{2}$ was to be analzyed using the TCD while $\mathrm{CO}$ and $\mathrm{CO}_{2}$ would be converted to $\mathrm{CH}_{4}$ in the methanizer and then analyzed using the FID. Operational problems with the methanizer have repeatedly plagued this approach. When high concentrations of $\mathrm{CO}$ and $\mathrm{CO}_{2}(\sim 10$ mol\%) were analyzed, the methanizer became overloaded and quickly failed. We continue to work with Shimadzu representatives on this problem, but so far without finding a solution.

An alternate analytical method involving argon carrier gas and using only the TCD has been developed. In this method we have also substituted a Poropack column for the Hayesep Q column used to separate $\mathrm{CO}_{2}$ from $\mathrm{H}_{2}, \mathrm{~N}_{2}$, and $\mathrm{CO}$. Although considerably simpler, the new method is less sensitive to low concentrations of $\mathrm{CO}$ and $\mathrm{CO}_{2}$ because of the similar thermal conductivities of these compounds and the argon carrier gas. The argon carrier gas provides the added advantage of permitting $\mathrm{N}_{2}$ to be added to the group of components which can be separated and analyzed.

A typical chromatogram using the new analytical method is shown in Figure 5. A calibration gas mixture containing $10 \% \mathrm{CO}, 10 \% \mathrm{CO}_{2}, 10 \% \mathrm{H}_{2}$, balance $\mathrm{N}_{2}$ was injected directly into the chromatograph in this test. $\mathrm{CO}_{2}$ was the first to pass through the TCD at 2.59 minutes, followed by $\mathrm{H}_{2}$ at 3.89 minutes, $\mathrm{N}_{2}$ at 4.83 minutes, and $\mathrm{CO}$ at 6.44 minutes.

The valve switching sequence used to achieve the above separation is illustrated in Figure 6 and described below. Normal operation is 


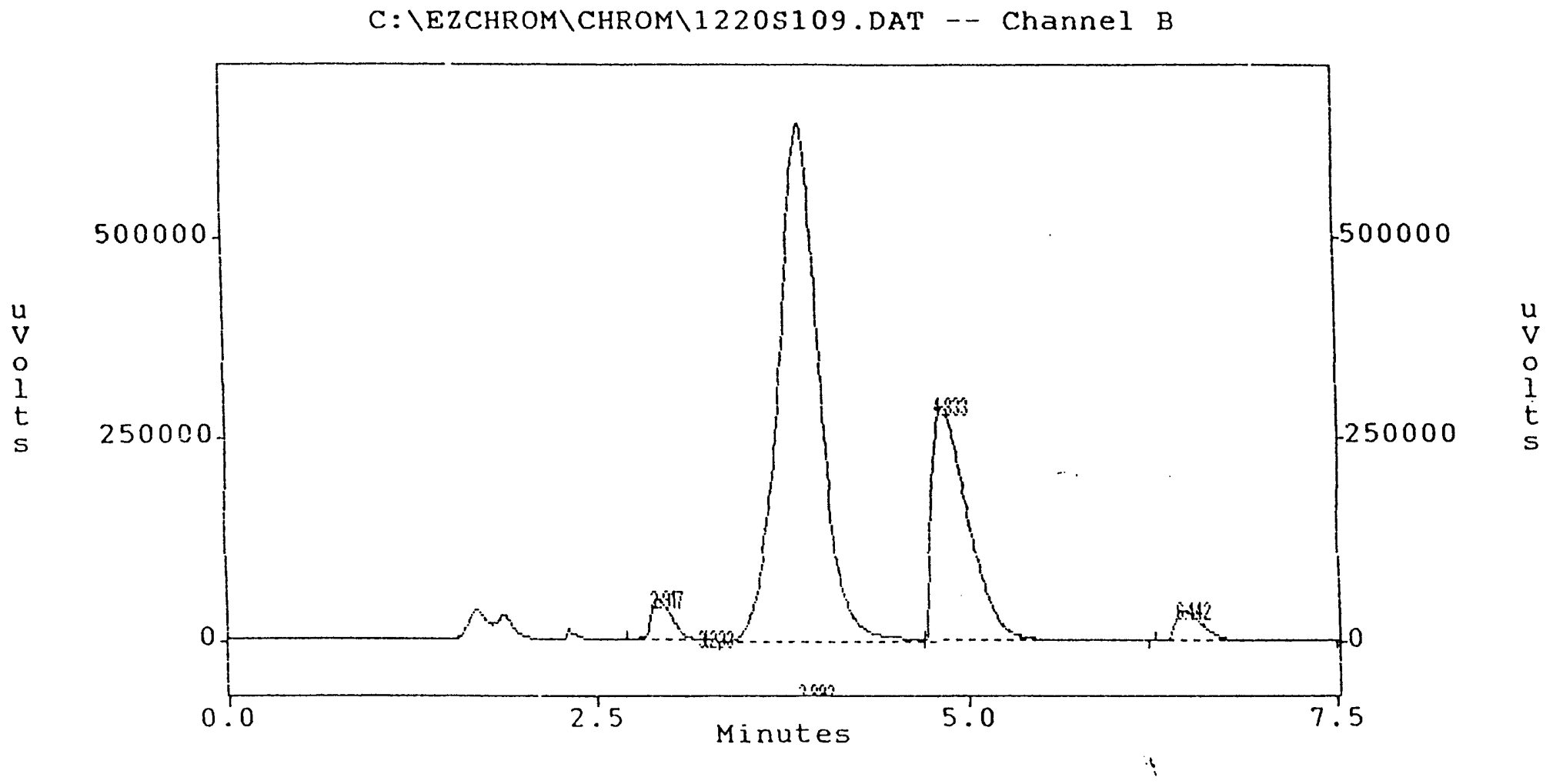

Channel B Report

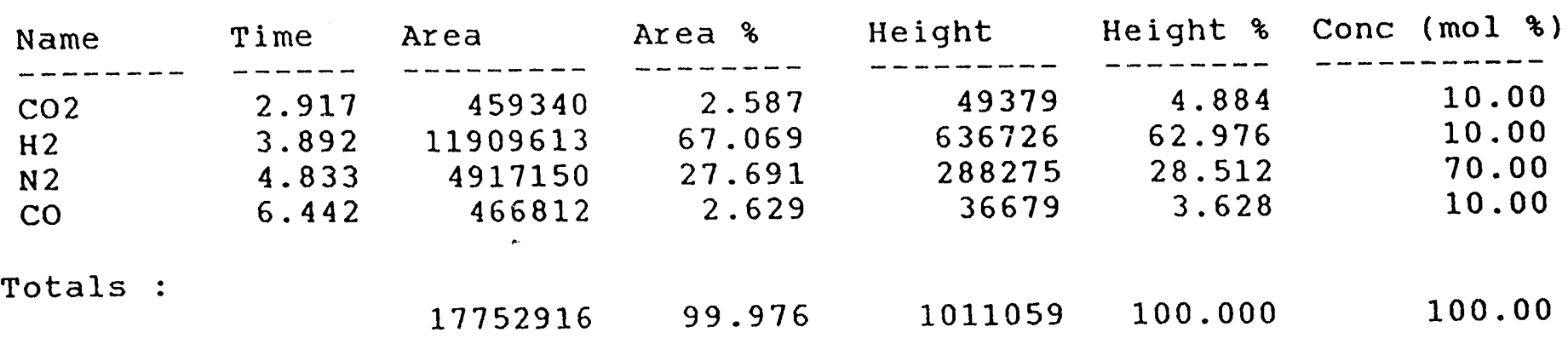

Figure 5. Chromatogram for Calibration Gas Standard 1. 

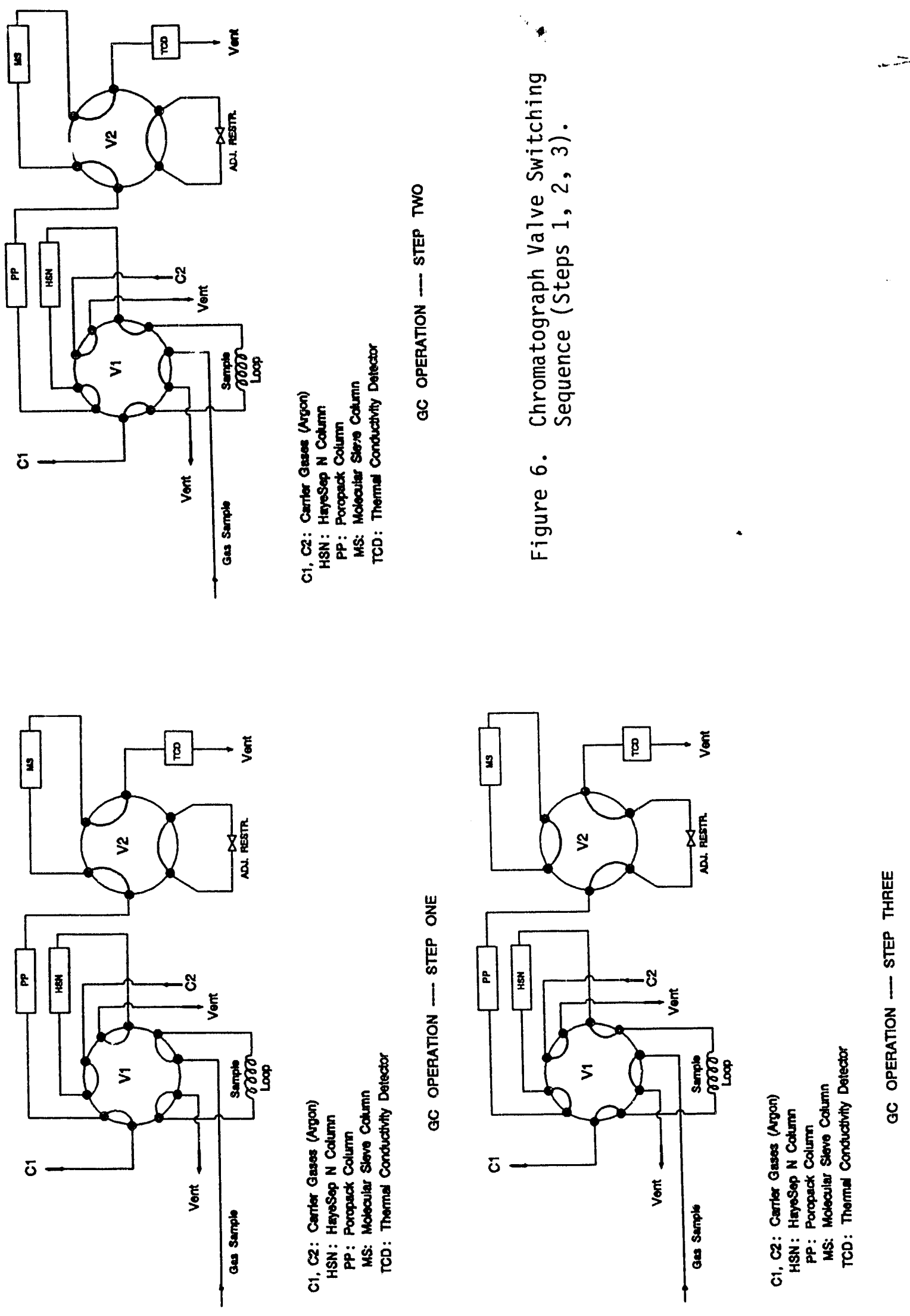


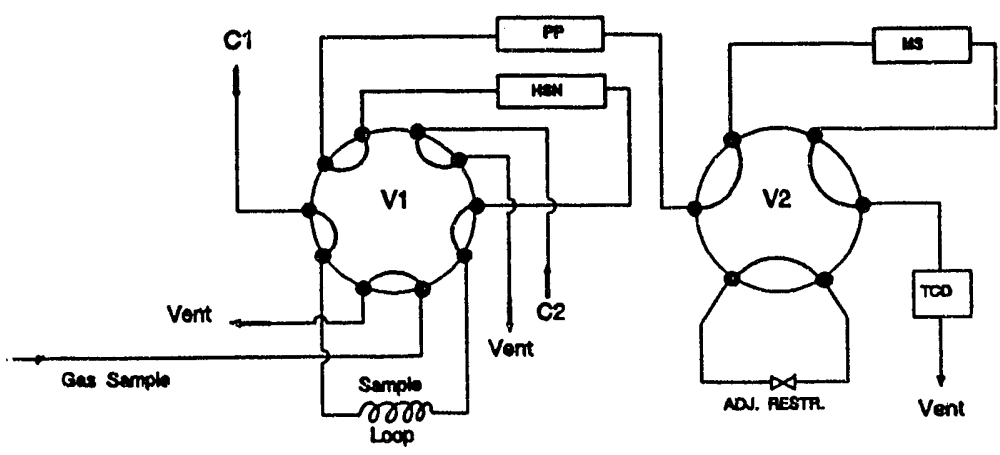

C1. C2: Carter Gases (Argon)

HSN: Hayosep N Cotumn

PP: Poropack Column

MS: Molecular Sieve Column

TCD: Therma Condictivity Detector

GC OPERATION - STEP FOUR

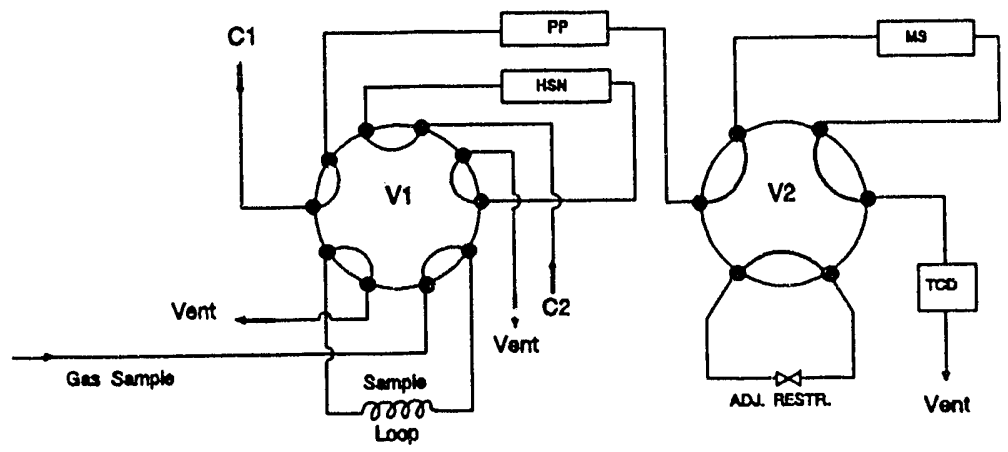

C1, C2: Carter Gases (Argon)

HSN : Hayesep N Column

PP: Poropack Column

MS: Molecular Slove Column

TCD: Thermed Conductivity Detector

GC OPERATION - STEP FIVE

Figure 6 (cont.). Chromatograph Valve Switching Sequence (Steps 4 and 5). 
illustrated as Step 1. The sample to be analyzed enters V1, flows through the sample loop, and exits to vent. Primary carrier (argon) enters V1 and flows, in turn, through the Poropack column, into V2, through the mol sieve column, back into V2, and exits through the TCD. Secondary carrier gas (also argon) enters V1, flows through the Hayesep $\mathrm{N}$ column, back into $\mathrm{V} 1$, and to vent.

Sampling is initiated at $t=0$ by switching $V 1$ to the position shown in step 2. The gas sample now enters $\mathrm{V} 1$ and exits directly to vent. Primary carrier picks up the sample contained in the sample loop and flows first to the Haysep $\mathrm{N}$ column where $\mathrm{H}_{2} \mathrm{O}$ is retained and the remaining gases -- $\mathrm{H}_{2}, \mathrm{~N}_{2}, \mathrm{CO}$, and $\mathrm{CO}_{2}-$ are carried to the Poropack column. The Hayesep $\mathbf{N}$ column is for future use as the samples presently being tested are $\mathrm{H}_{2} \mathrm{O}$-free. $\mathrm{CO}_{2}$ is retained on the Poropack column and $\mathrm{H}_{2}, \mathrm{~N}_{2}$, and $\mathrm{CO}$ pass to the mol sieve column for final separation. The primary carrier gas passes through the mol sieve and exits through the TCD. In this valve position secondary carrier gas flows directly into then out of V1 to vent.

After $\mathrm{H}_{2}, \mathrm{~N}_{2}$, and $\mathrm{CO}$ have been transferred to the mol sieve column, but before $\mathrm{CO}_{2}$ has eluted from the Poropack column, V2 is switched to the position shown in step 3. This switch occurred at 2.30 minutes in the present example. The gas sample continues to flow into V1 and directly out of V1 to vent. Primary carrier flows into V1, through the Poropack column where $\mathrm{CO}_{2}$ is eluted, through the adjustable restrictor arm of V2, and then through the TCD. $\mathrm{H}_{2}, \mathrm{~N}_{2}$, and $\mathrm{CO}$ adsorbed on the mol sieve column are isolated from carrier gas flow during this time. Secondary carrier gas continues to flow into and out of v1 to vent.

At an appropriate time after $\mathrm{CO}_{2}$ has passed through the TCD, $t=3.3$ minutes in this example, $v 2$ is switched back to its original 
position as shown in step 4. The gas sample continues to flow into and out of V1 to vent. The primary carrier gas flow path is through V1, the Poropack column, into $\mathrm{V} 2$, the mol sieve column, back to $\mathrm{V} 2$, and then the TCD in sequence. During this period final separation of $\mathrm{H}_{2}, \mathrm{~N}_{2}$, and CO occurs on the mol sieve column and these components pass through the TCD at the times shown in Figure 5. Secondary carrier gas continues to flow into and out of $\mathrm{VI}$ to vent.

In the final switching step, at $t=5.0$ minutes, $V 1$ is returned to its original position (Step 5). The gas sample once again flows into V1, through the sample loop, back into V1, and to vent. Primary carrier gas flows through the Poropack and mol sieve columns and through the TCD to vent. Note from Figure 5 that this switch occurs while $\mathrm{N}_{2}$ and $\mathrm{CO}$ are still retained on the mol sieve. Secondary carrier gas flows into V1 and through the Hayesep $\mathrm{N}$ column to vent. The secondary carrier flow direction through the Hayesep $\mathbf{N}$ column is in the opposite direction to the primary carrier gas flow direction so that water can be back-flushed from the column during this period.

Valve positions in Steps 1 and 5 are identical so that the sample sequence can be started over at any time after the CO has eluted from the mol sieve column and water has been backflushed from the Hayesep $\mathrm{N}$ column. Overall cycle times in the range of 7.5 to 10 minutes appear reasonable.

Additional adjustments in the timing sequence may be necessary when samples containing $\mathrm{H}_{2} \mathrm{O}$ are analyzed. In particular, the timing of Step 5 must be such that $\mathrm{H}_{2} \mathrm{O}$ is not allowed to elute from the Hayesep $\mathrm{N}$ onto the Poropack column.

Calibration tests using the gas mixture compositions shown in Table I were carried out. Results in the form of peak area versus mol 
Table I. Calibration Gas Compositions (mol \%).

$\begin{array}{cccc} & & \text { Standard } & \\ \text { Component } & 1 & 2 & 3 \\ \mathrm{CO} & 10.0 & 0.05 & 1.0 \\ \mathrm{CO}_{2} & 10.0 & 0.10 & 4.0 \\ \mathrm{H}_{2} & 10.0 & 15.0 & 20.0 \\ \mathrm{~N}_{2} & 70.0 & 84.85 & 75.0\end{array}$


fraction for the four components are shown in Figure 7 . The response is quite linear for $\mathrm{CO}, \mathrm{CO}_{2}$, and $\mathrm{N}_{2}$ while the $\mathrm{H}_{2}$ response is clearly nonlinear. Chromatograph software is capable of correlating the calibration data in three ways:

(1) a single best-fit straight line through all calibration points;

(2) separate straight lines through successive calibration points; and,

(3) a quadratic best-fit line through all calibration points.

Because of the obvious nonlinearity in the $\mathrm{H}_{2}$ response, the quadratic calibration will be used. The quadratic constants determined from the calibration gas analyses are summarized in Table II.

This calibration method produces absolute response factors which means that the sum of the mol fractions resulting from the analysis of a gas of unknown composition is not necessarily 1.0. The implications will become clear in the following section when results of the initial calcination test are presented.

The analytical procedure described above will be used for gas analyses for now. However, further improvements will be sought. Three problems or potential problems are presently known. First, as previously described, the TCD does not have the desired sensitivity for low concentrations of $\mathrm{CO}$ and $\mathrm{CO}_{2}$. The lowest concentration in the calibration gases was $0.05 \mathrm{~mol} \% \mathrm{CO}$ (500 ppm volume), and this analysis pushed the sensitivity limits of the TCD. We prefer to be able to analyze $\mathrm{CO}$ and $\mathrm{CO}_{2}$ concentrations to the 50 or $100 \mathrm{ppm}$ level. Secondly, the nonlinearity in the $\mathrm{H}_{2}$ response will be investigated. Since a linear response was expected, we must be convinced that the nonlinear 

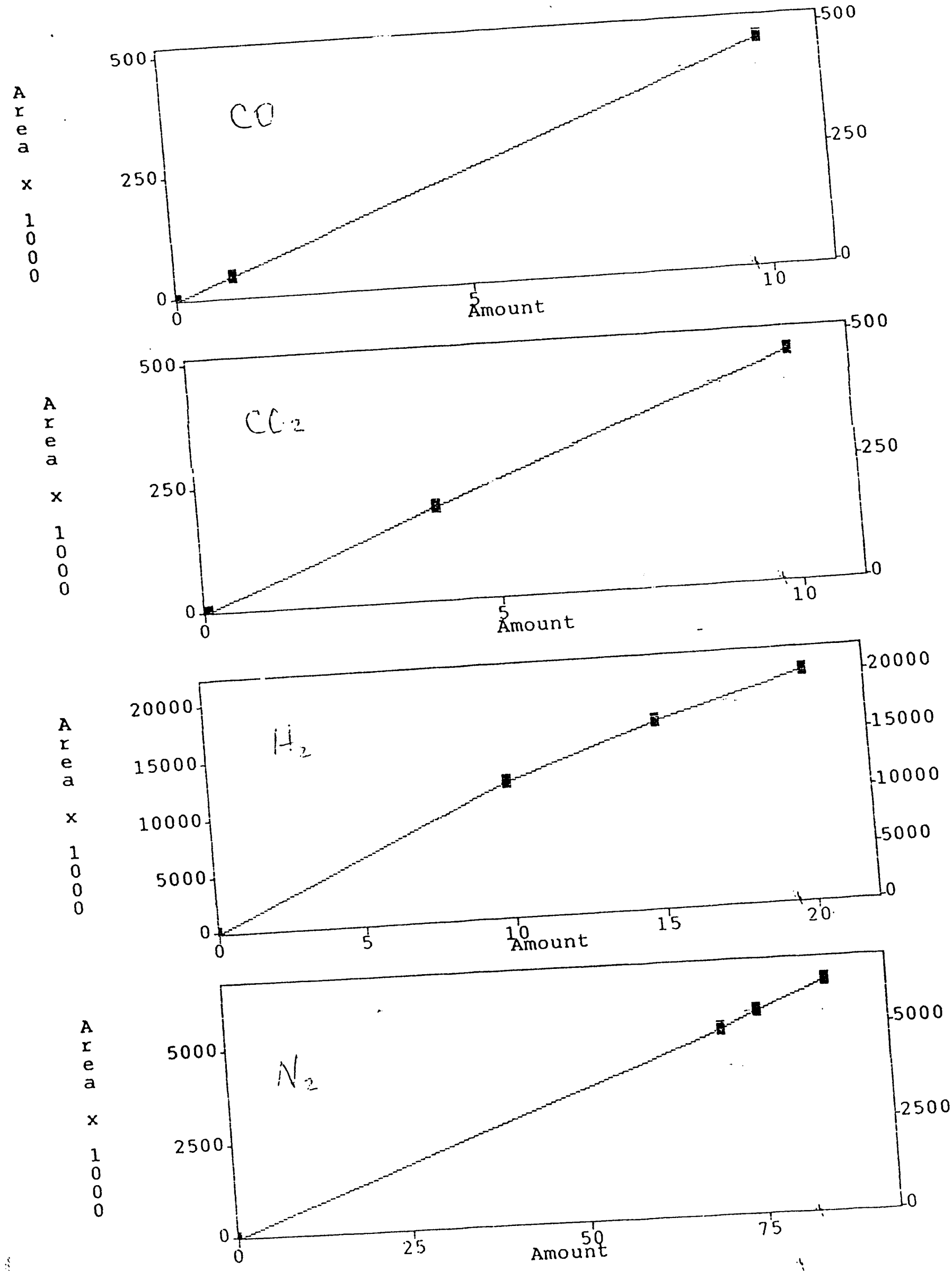

Figure 7. Chromatograph Responses for Three Calibration Gas Compositians. 
Table II. Quadratic Constants for Chromatograph Calibration.

$$
\begin{aligned}
y_{i} & =a_{i} A_{i}+b_{i} A_{i}^{2} \\
y_{i} & =\text { mol percent, component } i \\
a_{i}, b_{i} & =\text { calibration constants } \\
A_{1} & =\text { chromatogram peak area } \\
R_{i}^{2} & =\text { correlation coefficient }
\end{aligned}
$$

$\begin{array}{cccc}\text { Component } & \mathrm{a}_{\mathrm{i}} & \mathrm{b}_{\mathrm{i}} & \mathrm{R}_{\mathrm{i}}^{2} \\ \mathrm{CO} & 2.125 \times 10^{-5} & 3.656 \times 10^{-13} & 1.0000 \\ \mathrm{CO}_{2} & 1.994 \times 10^{-5} & 3.977 \times 10^{-12} & 1.0007 \\ \mathrm{H}_{2} & 6.015 \times 10^{-7} & 1.957 \times 10^{-14} & 1.0006 \\ \mathrm{~N}_{2} & 1.536 \times 10^{-5} & -2.463 \times 10^{-13} & 1.0000\end{array}$


response is real. Finally, there is a potential problem, not discussed explicitly in this report, associated with an unsteady-state $\mathrm{CO}_{2}$ response. For example, when a calibration gas with low $\mathrm{CO}_{2}$ concentration is analyzed immediately following the analysis of a gas with high $\mathrm{CO}_{2}$ concentration, several injections may be required before the TCD response reaches a constant value. It was this concern that led to the elimination of the mol sieve filter in the product gas line (see previous discussion).

\section{INITIAL CALCINATION TEST RESULTS}

In the first actual test of the system under reaction conditions, dolomite (sorbent 9) was calcined at $750^{\circ} \mathrm{C}$ and 15 atm using a constant flow rate of $\mathrm{N}_{2}$. The furnace temperature was programmed to increase at a rate of $5^{\circ} \mathrm{C} / \mathrm{min}$ to $750^{\circ} \mathrm{C}$ and to remain isothermal thereafter. Actual reactor temperatures are shown in Figure 8 . Other than the difference in the final temperature, the response shown in Figures 3 and 8 are similar.

Product gas samples were collected and analyzed every 10 minutes with the first sample taken 10 minutes after heating was initiated. Product gas composition was determined on the basis of the calibrations from Figure 7. A detailed summary of product gas composition as a function of time is presented in Table III while Figure 9 shows the mol fraction $\mathrm{CO}_{2}$ in the calcination product gas as a function of time.

In the first seven samples the calcination product gas contained nothing but $\mathrm{N}_{2}$. First traces of $\mathrm{CO}_{2}$ appeared in the 80 minute sample when the sorbent temperature was about $300^{\circ} \mathrm{C}$. The mol fraction $\mathrm{CO}_{2}$ remained equal to or less than 0.002 through sample 11 at 110 minutes, at which time the sorbent temperature had increased to about $500^{\circ} \mathrm{C}$. 


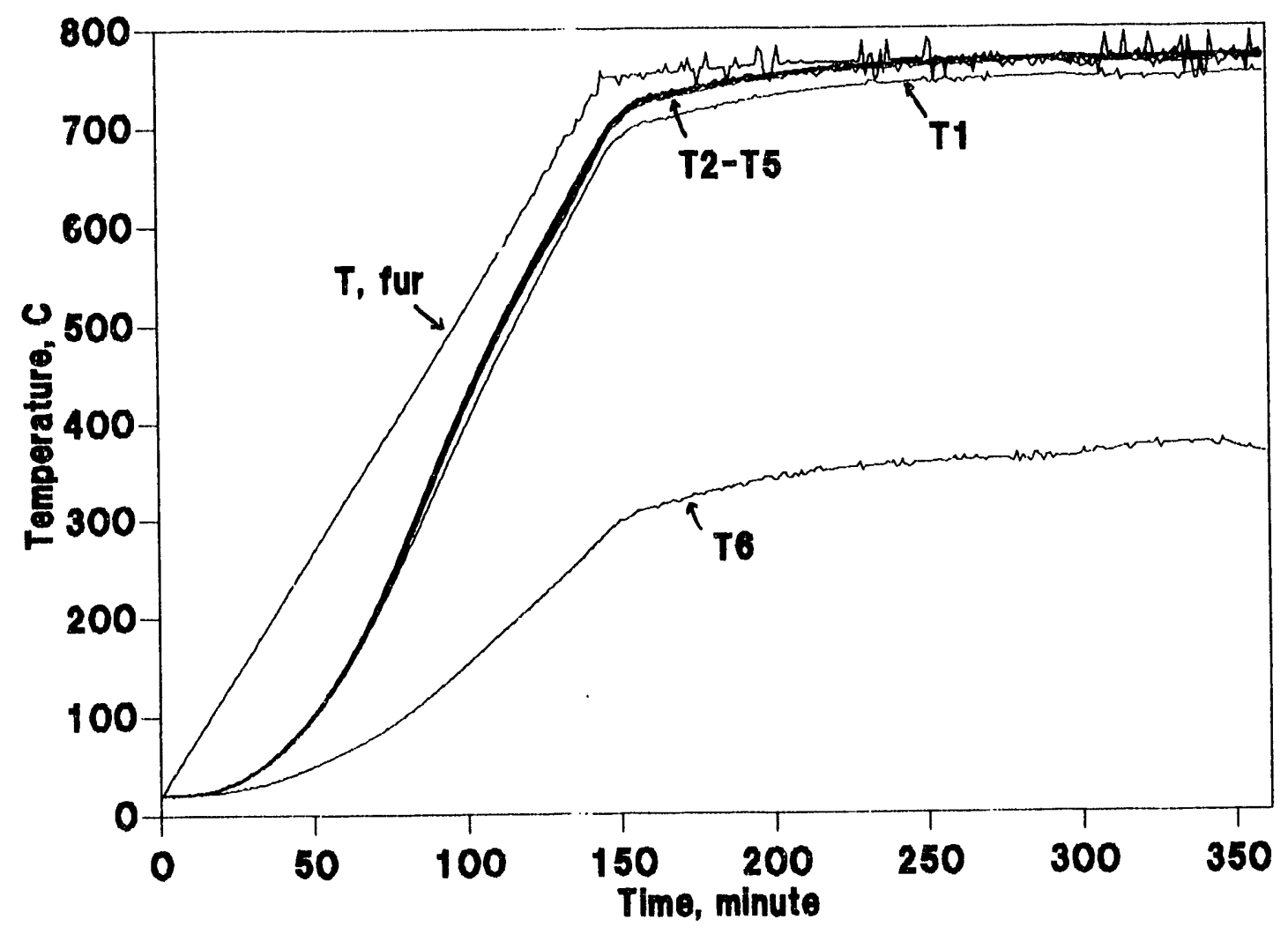

Figure 8. Reactor Temperature-Time History During Initial Calcination Test. 
Table III. Product Gas Composition During Calcination Test.

\begin{tabular}{|c|c|c|c|c|}
\hline \multirow[b]{2}{*}{ Time (min) } & \multicolumn{4}{|c|}{ Composition, mol告 } \\
\hline & $\mathrm{CO}_{2}$ & $\mathrm{~N}_{2}$ & $\mathrm{CO}$ & Total \\
\hline 10 & 0 & 96.5 & 0 & 96.5 \\
\hline 20 & 0 & 96.2 & 0 & 96.2 \\
\hline 30 & 0 & 96.2 & 0 & 96.2 \\
\hline 40 & 0 & 94.0 & 0 & 94.0 \\
\hline 50 & 0 & 97.9 & 0 & 97.9 \\
\hline 60 & 0 & 95.9 & 0 & 95.9 \\
\hline 70 & 0 & 97.2 & 0 & 97.2 \\
\hline 80 & $<0.1$ & 94.8 & 0 & 94.8 \\
\hline 90 & $<0.1$ & 95.7 & 0 & 95.7 \\
\hline 100 & $<0.1$ & 97.1 & 0 & 97.1 \\
\hline 110 & 0.2 & 95.4 & 0 & 95.6 \\
\hline 120 & 3.4 & 92.9 & $<0.1$ & 96.3 \\
\hline 130 & 14.9 & 84.4 & 0.2 & 99.5 \\
\hline 140 & 28.6 & 70.6 & 0.4 & 99.6 \\
\hline 150 & 27.4 & 70.7 & 0.6 & 98.7 \\
\hline 160 & 26.1 & 70.0 & 0.8 & 96.9 \\
\hline 170 & 2.4 & 94.0 & 0.3 & 96.7 \\
\hline 180 & 1.5 & 91.6 & 0.2 & 93.3 \\
\hline 190 & 1.6 & 96.6 & 0.2 & 98.4 \\
\hline 200 & 1.7 & 95.6 & 0.2 & 97.5 \\
\hline 210 & 1.8 & 92.7 & 0.2 & 94.7 \\
\hline 220 & 1.9 & 93.9 & 0.2 & 96.0 \\
\hline 230 & 2.0 & 95.8 & 0.2 & 98.0 \\
\hline 240 & 2.0 & 94.8 & 0.2 & 97.0 \\
\hline 250 & 2.1 & 92.4 & $0 . .2$ & 94.7 \\
\hline 260 & 2.1 & 96.7 & 0.2 & 99.0 \\
\hline 270 & 2.2 & 97.6 & 0.2 & 100.0 \\
\hline 280 & 2.1 & 95.1 & 0.2 & 97.4 \\
\hline 290 & 2.1 & 98.4 & 0.2 & 100.7 \\
\hline 300 & 2.2 & 96.4 & 0.2 & 98.8 \\
\hline 310 & 2.1 & 100.6 & 0.1 & 102.8 \\
\hline 320 & 2.0 & 106.9 & 0.1 & 109.0 \\
\hline 330 & 2.0 & 108.7 & $<0.1$ & 110.7 \\
\hline 340 & 2.0 & 108.4 & $<0.1$ & 110.4 \\
\hline 350 & 2.0 & 108.0 & $<0.1$ & 110.0 \\
\hline 360 & 2.1 & 97.1 & 0.1 & 99.3 \\
\hline 370 & 2.0 & 99.4 & 0.1 & 101.5 \\
\hline 380 & 2.4 & 97.2 & 0.1 & 99.7 \\
\hline 390 & 3.9 & 96.7 & 0.2 & 100.8 \\
\hline 400 & 3.9 & 97.9 & 0.2 & 102.0 \\
\hline
\end{tabular}




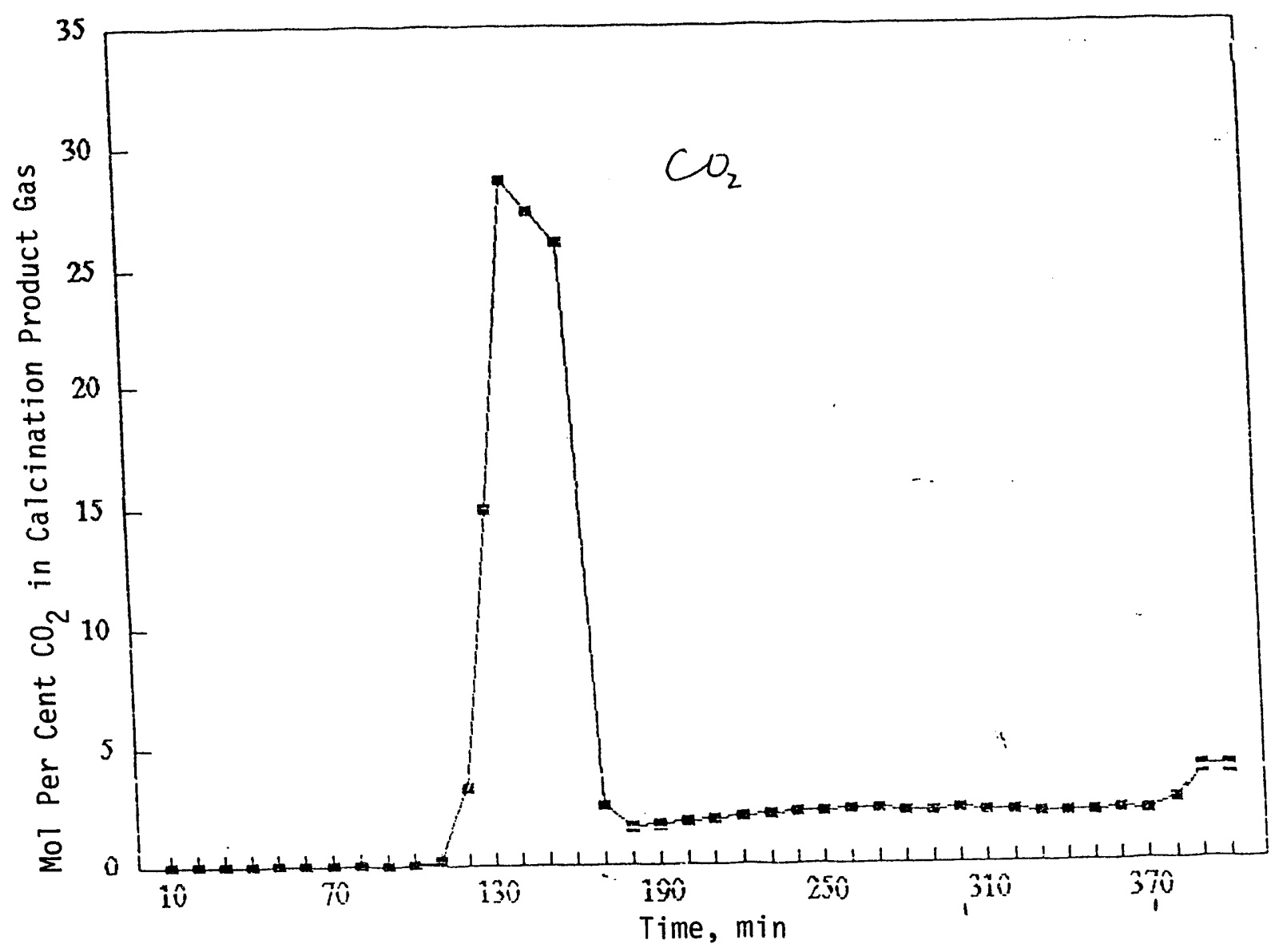

Figure 9. Results of Initial Calcination Test Showing Mol Per Cent $\mathrm{CO}_{2}$ in Product Gas as a Function of Time. 
Thereafter, the $\mathrm{CO}_{2}$ mol fraction increased quickly and reached a maximum of 0.286 at 140 minutes and a temperature of about. $680^{\circ} \mathrm{C}$. The mol fraction of $\mathrm{CO}_{2}$ remained high in samples 15 and 16 (150 and 160 minutes) by which time the temperature had reached about $720^{\circ} \mathrm{C}$. The $\mathrm{CO}_{2}$ mol fraction then decreased quickly and remained in the range of 0.015 to 0.024 through 300 minutes. The relative constancy of the $\mathrm{CO}_{2}$ concentration during this period suggests that the final stages of calcination are quite slow. However, the failure of the $\mathrm{CO}_{2}$ concentration to return to zero could also be caused by gradual desorption of $\mathrm{CO}_{2}$ from reactor surfaces or from the presence of dead volume within the reactor. As previously mentioned, the mol sieve filter was removed from the product line because it was thought to be a possible adsorbent for $\mathrm{CO}_{2} \cdot$ Similarly, the condenser might serve as a source of dead volume. These potential problems will be investigated in the future.

After sample 30 (300 minutes) the $\mathrm{N}_{2}$ flow rate was increased but without apparent effect on the $\mathrm{CO}_{2}$ concentration in the product gas. Finally, after sample 36 (360 minutes) the reactor temperature set-point was increased from 750 to $800^{\circ} \mathrm{C}$. The increase in the $\mathrm{CO}_{2}$ concentration after 390 minutes is consistent with the interpretation that the final stages of calcination are quite slow. The test was terminated after 400 minutes.

An unexpected result shown in Table III was the appearance of relatively small quantities of $\mathrm{CO}$ in the calcination product gas. CO first appeared in sample $12(120$ minutes) at a temperature of approximately $550^{\circ} \mathrm{C}$. The $\mathrm{CO}$ mole fraction peaked at 0.008 in sample 16 (160 minutes) at $\mathrm{T} \sim 720^{\circ} \mathrm{C}$, and quickly declined to the 0.001 to 0.002 
level, but never disappeared completely. CO was not expected since both $\mathrm{CaCO}_{3}$ and $\mathrm{MgCO}_{3}$ should decompose clearly to $\mathrm{CaO}, \mathrm{MgO}$, and $\mathrm{CO}_{2}$. The probable source of $\mathrm{CO}$ is the reaction

$$
\mathrm{C}(\mathrm{s})+\mathrm{CO}_{2}(\mathrm{~g}) \rightarrow 2 \mathrm{CO}(\mathrm{g})
$$

with the reactor tube walls serving as the carbon source.

The last column in Table III shows the total mol percent of $\left(\mathrm{CO}_{2}+\right.$ $\mathrm{N}_{2}+\mathrm{CO}$ ) determined using the quadratic calibration factors from Table II. Recall that since the calibration factors are absolute, the sum of the mol per cents do not necessarily equal 100. In this test the sum ranged from a low of $93.3 \%$ to a high of $110.7 \%$.

Although a number of questions were raised by the initial calcination test, on a overall basis we consider it to be a success. All components of the reactor system functioned woll, and many of the questions will be answered as additional runs are compieted. 

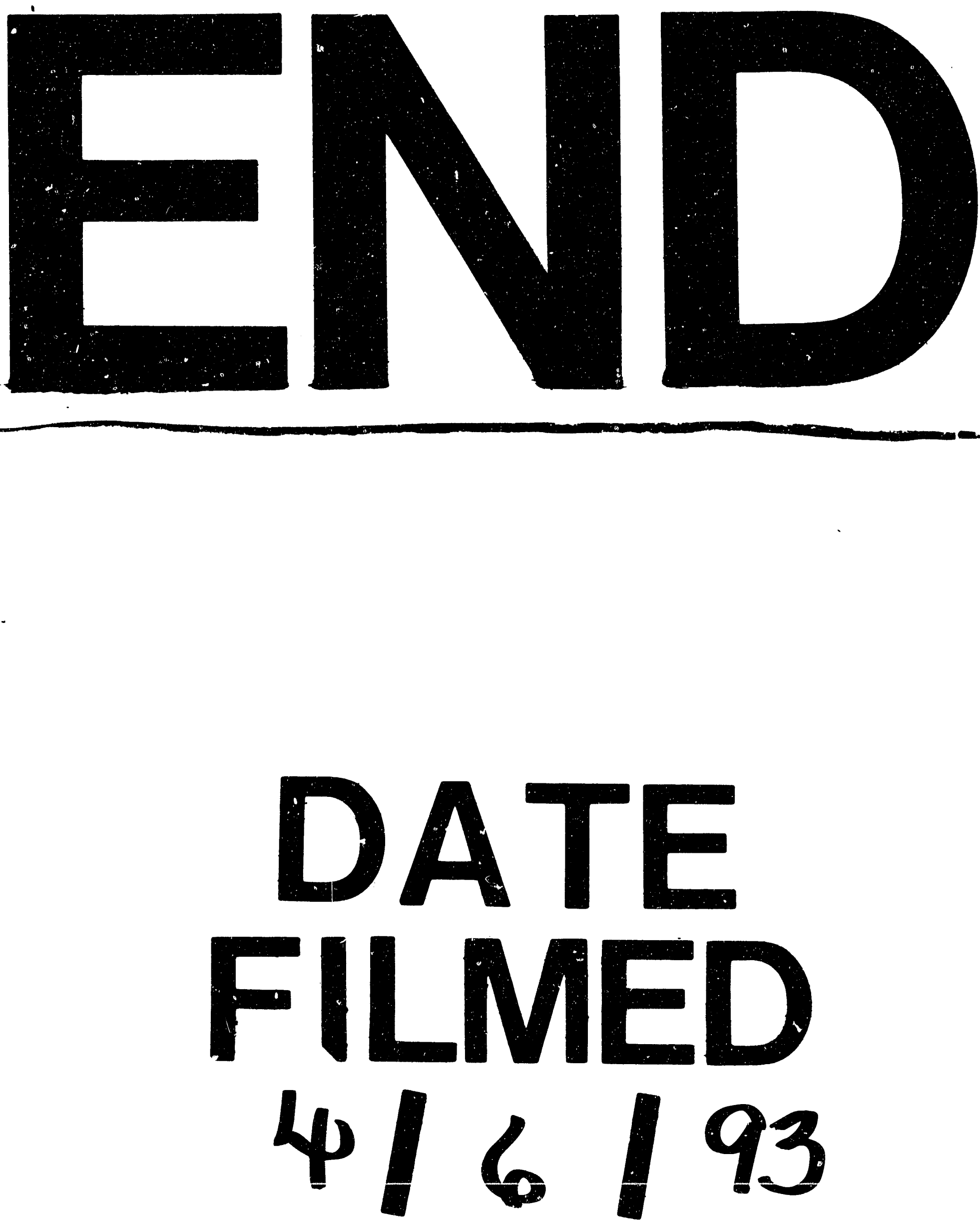
